\title{
Incomplete surgical staging in clinical early-stage ovarian cancer: guidelines versus daily practice
}

\section{P Laven, MD ${ }^{\mathrm{a}, 1, *}$, JJ Beltman, MD, PhD ${ }^{\mathrm{b}, 1}$, JE Bense, MD ${ }^{\mathrm{b}}$, MA van der Aa, PhD ${ }^{\mathrm{c}}, \mathrm{T}_{\text {Van }}$ Gorp, MD, PhD ${ }^{\mathrm{a}, \mathrm{d}, 2}$,} MC Vos, MD, PhD ${ }^{\mathrm{e}}$, D Boll, MD, PhD ${ }^{\mathrm{f}}$, HGJ Arts, MD, PhD ${ }^{\mathrm{g}}, \mathrm{N}^{\mathrm{R}}$ Reesink, MD, PhD ${ }^{\mathrm{h}}$, JB Trimbos, MD, PhD ${ }^{\text {b }}$, RFPM Kruitwagen, MD, PhD ${ }^{\text {a }}$

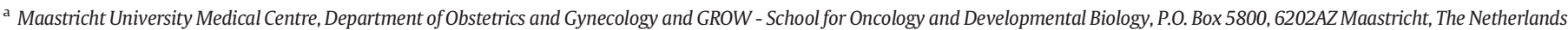

${ }^{\mathrm{b}}$ Leiden University Medical Centre, Department of Obstetrics and Gynecology, Leiden, The Netherlands

${ }^{\mathrm{c}}$ Netherlands Comprehensive Cancer Organization, Department of Research, Utrecht, The Netherlands

d Department of Obstetrics and Gynecology, Division of Gynecological Oncology, Leuven Cancer Institute, University Hospitals Leuven, Leuven, Belgium

e Elisabeth-TweeSteden Hospital, Department of Obstetrics and Gynecology, Tilburg, The Netherlands

f Catharina Cancer Institute, Eindhoven, Department of Obstetrics and Gynecology, Eindhoven, The Netherlands

${ }^{g}$ University Medical Centre Groningen, Department of Obstetrics and Gynecology, Groningen, The Netherlands

${ }^{\mathrm{h}}$ Hospital Medical Spectrum Twente, Department of Obstetrics and Gynecology, Enschede, The Netherlands
}

\section{A R T I C L E I N F O}

\section{Article history:}

Received 25 May 2021

Received in revised form 8 September 2021

Accepted 16 September 2021

Available online 14 October 2021

\section{Keywords:}

Ovarian cancer

Surgical oncology

Staging

Lymph nodes

\begin{abstract}
A B S T R A C T
Background: Incomplete surgical staging of patients with early-stage epithelial ovarian cancer (EOC) has been reported in up to $98 \%$ of cases, when based on the International Federation of Obstetrics and Gynecology (FIGO) staging procedure. The aim of the present retrospective study was to clarify the reasons for incomplete staging. Methods: The PRISMA (Prevention Recovery Information System for Monitoring and Analysis) technique was used to evaluate cases with FIGO I-IIa EOC based on incomplete staging from five gynecologic oncologic center hospitals in the Netherlands in the period 2010-2014.

Results: Fifty cases with an incomplete surgical staging of EOC according to national guidelines were included. The most common reasons for incomplete staging were insufficient random biopsies of the peritoneum $(\mathrm{n}=34$, $68 \%)$, and less than ten lymph nodes being resected and/or found at pathology ( $n=16,32 \%)$. The most mentioned reason for not performing biopsies was, besides forgetting to do so, believing that after careful inspection and palpation, taking biopsies is irrelevant and/or already are being taken while performing a hysterectomy (peritoneum of cul-de-sac, bladder). The value of contralateral pelvic lymph node dissection in case of a unilateral ovarian malignancy was also doubted, influencing the number of lymph nodes resected.

Conclusions: The most important reasons for incomplete staging in EOC are, besides omitting elements by accident, questioning the importance of obligatory elements of the staging procedure. A structured list of staging steps during surgery and more evidence-based consensus concerning these obligatory elements might increase the number of complete staging procedures in EOC.
\end{abstract}

(C) 2021 Published by Elsevier Inc. This is an open access article under the CC BY-NC-ND license (http:// creativecommons.org/licenses/by-nc-nd/4.0/).

\footnotetext{
* Corresponding author at: Department of Obstetrics and Gynecology, Maastricht University Medical Centre, P.O. Box 5800, 6202AZ Maastricht, The Netherlands.

E-mail addresses: p.laven@mumc.nl (P. Laven), j.j.beltman@lumc.nl (J.J. Beltman), joellbense@gmail.com (J.E. Bense), M.vanderAa@iknl.nl (M.A. van der Aa), toon.vangorp@uzleuven.be (T. Van Gorp),c.vos@etz.nl (M.C. Vos), bolldorry@gmail.com (D. Boll), h.j.g.arts@umcg.nl (H.G.J. Arts), n.reesink-peters@mst.nl (N. Reesink), trimbos@gmail.com (J.B. Trimbos), r.kruitwagen@mumc.nl (R.F.P.M. Kruitwagen).

${ }^{1}$ Both authors contributed equally to this manuscript.

2 At present: Department of Obstetrics and Gynecology, Division of Gynecological Oncology, Leuven Cancer Institute, University Hospitals Leuven, Leuven, Belgium.
}

\section{Introduction}

In newly diagnosed clinical early-stage epithelial ovarian cancer (EOC), the aim of surgery is to resect the tumor combined with an adequate staging procedure. This will provide prognostic information by means of the definite stage and will define whether adjuvant chemotherapy is needed or can be safely omitted [1]. According to the International Federation of Obstetrics and Gynecology (FIGO) a staging procedure should include a peritoneal washing or a sample of ascites, hysterectomy and bilateral oophorectomy, infracolic omentectomy (complete, partial or biopsies), numerous peritoneal biopsies from several locations at risk for tumor implantation, pelvic and para-aortic lymphadenectomy and biopsies of suspected lesions and adhesions 
[2]. The Dutch guideline has incorporated these staging elements with the adjustment that overall, at least 10 lymph nodes should be resected from specific locations in the pelvic and paraaortic area.

Despite the FIGO recommendation, incomplete surgical staging has been reported in up to $98 \%$ of cases in different studies [3-8]. Studies examining the causes of incomplete staging are scarce and often based on assumptions such as lack of specialized skills and/or knowledge of the gynecologist $[3,5,6]$. An example of such an assumption is the opinion that after careful inspection taking biopsies of macroscopically normal peritoneum is irrelevant. [8]

The Prevention Recovery Information System for Monitoring and Analysis (PRISMA) was originally developed to manage human error in the chemical process industry, but has also been applied in healthcare (PRISMA-Medical) [9-11]. The main goal of PRISMA is to build a quantitative database of incidents and process deviations, in order to facilitate the development and evaluation of system-based preventive strategies. The PRISMA-Medical method consists of a causal tree incident description followed by a classification of root causes. Causal trees are used because nearly all incidents have more than one cause. By continuing to ask 'why' of each event (beginning with the top event), a structure of causes and consequences arises, until the root causes are identified at the bottom of the tree. These root causes are subsequently classified by linking them to one of the categories. As both active failures (human error) and latent conditions (technical and organizational failures) surrounding incidents are systematically considered with the PRISMA-Medical method, the results of this analysis can be used to provide a more realistic view of daily practice [10]. In this study, we used PRISMA to study the causes of incomplete staging in EOC in detail instead of making assumptions based on epidemiological data.

\section{Material and methods}

Five hospitals participated in the study and all hospitals fulfilled the Dutch criteria to be a gynecologic oncology center within their region among which the performance of 20 or more ovarian cancer debulking procedures each year. All surgery was performed by or under supervision of qualified gynecologic oncologists. Patients with EOC FIGO stage I - Ila undergoing a staging procedure in the period from 2010 until 2014 were included if one of the following incidences had occurred: no sampling of ascites or peritoneal washing; less than five peritoneal biopsies resection; less than ten lymph nodes; no high para-aortal, ipsi- and/or contralateral pelvic lymph nodes; no omentum biopsy or resection. Patients in whom a staging procedure was waived in advance (i.e. because of age, comorbidities) were excluded.

Within the period 2014-2015, cases were selected retrospectively from the Netherlands Cancer Registry (NCR). The NCR is maintained by the Netherlands Comprehensive Cancer Organization and contains data of all cancer patients in the Netherlands. The NCR, which reached full national coverage in 1989, relies on notification of all newly diagnosed malignancies via the automated nationwide pathology archive. Trained registrars use standardized forms to collect patient information from medical records, and regular consistency checks are performed to ensure the quality of the data held in the NCR. Information on date of death were obtained from the municipal demography registries.

The following data were examined in this study: age; histological subtype; tumor stage; differentiation grade; inspection and palpation of all peritoneal surfaces; biopsies of any suspect lesions for metastases; biopsies or removal of any adhesions surrounding the primary tumor; peritoneal washing or ascites; infracolic omentectomy or biopsy; biopsies of peritoneum of the bladder, cul-de-sac, pelvic sidewalls, ovarian fossa, right and left paracolic gutter, and right hemi-diaphragm; number of lymph nodes removed; regions of lymph nodes removed (high paraaortal / paracaval, ipsilateral pelvic, contralateral pelvic). In 2020 the 5years survival was added to the data.
The PRISMA commission consisted of eight gynecologists, a member of the NCR (MA), and a medical researcher. All included cases were analyzed and discussed with the gynecologic oncologists of each participating hospital in the period 2015-2016. All members participating in the commission signed a non-disclosure agreement.

\section{Results}

Study population. Fig. 1 shows the study population and reasons for exclusion. Fifty-eight patients were selected by the NCR data managers. One selected patient had advanced stage disease at pathology (IIIA1 based on a lymph node metastasis) and was excluded. After analyzing and discussing the patients with the gynecologists of each participating hospital, seven patients (12\%) appeared to have had a complete staging after all, but were interpreted differently during selection by the NCR data managers. In two of these cases the location of the aortocaval lymph node dissection was not well described, and in five cases the gynecologist used anatomical terms describing the location, that were unfamiliar to the data managers. Therewith, 50 cases remained for inclusion in this study.

Most patients ( $n=34,68 \%$ ) underwent one operation, all laparotomies with frozen section. In 15 patients (30\%) a second operation was performed (of which laparoscopic in 10 patients), and in one patient (2\%) despite three surgical procedures (two laparotomies and one laparoscopy) the staging procedure still was incomplete.

Nineteen patients (38\%) received adjuvant chemotherapy. In two cases, it was unknown whether adjuvant chemotherapy was given (4\%) and one patient refused chemotherapy. For the remaining 29 patients (58\%), according to the gynecologic oncologist, there was no indication for adjuvant chemotherapy because the FIGO stage was based on a complete staging procedure.

Incomplete staging. The number of missing elements was one in $12 \mathrm{pa}-$ tients (24\%), two in 17 patients (34\%), three in eight patients (16\%), four in four patients (8\%), five in four patients (8\%), and six or more in the remaining five patients (10\%).

Table 1 shows incompleteness of the staging procedure with respect to the different elements. The most frequent reason for incomplete staging was insufficient peritoneal biopsies of the different locations $(n=34$; $68 \%$ ). Reasons for not performing certain biopsies were 1) simply forgetting to do so $(\mathrm{n}=11), 2$ ) presumption that the biopsy (cul-de-sac and bladder peritoneum) is already part of the hysterectomy $(n=5), 3)$ the opinion of the surgeon that after careful inspection taking biopsies of macroscopically normal peritoneum is irrelevant $(n=6)$, 4) chemotherapy already being indicated (i.e. based on peroperative findings such as rigid adhesions of the ovarian tumor to the pelvic wall), hence complete staging would not be contributory $(n=6), 5)$ technical problems $(n=3)$,

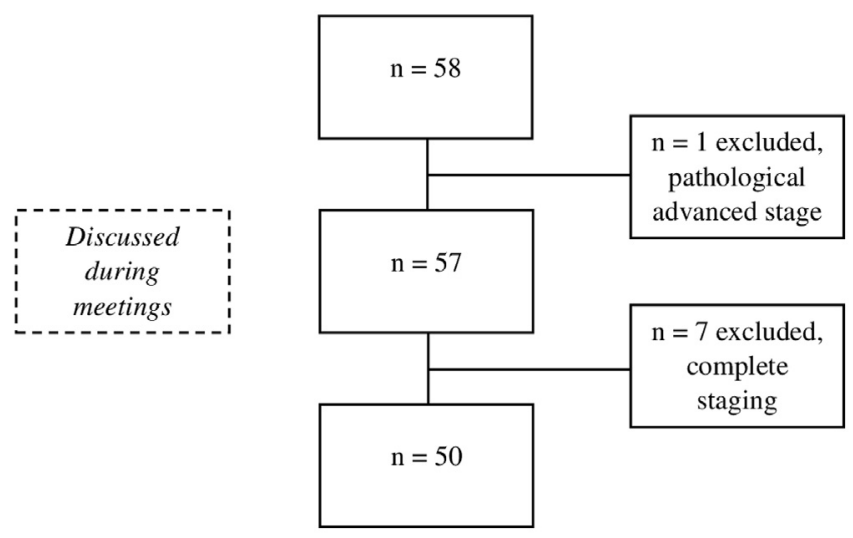

Fig. 1. Flowchart inclusion. 
Table 1

Incompleteness of the staging procedure per element.

\begin{tabular}{llll}
\hline $\begin{array}{l}\text { Part of staging } \\
\text { procedure }\end{array}$ & $\begin{array}{l}\mathrm{n}(\%) \\
\text { Complete }\end{array}$ & $\begin{array}{l}\mathrm{n}(\%) \\
\text { Incomplete }\end{array}$ & $\begin{array}{l}\mathrm{n}(\%) \\
\text { Inapplicable }\end{array}$ \\
\hline Inspection & $50(100 \%)$ & $0(0 \%)$ & \\
Palpation & $43(86 \%)$ & $7(14 \%)$ & \\
Ascites & $39(76 \%)$ & $12(24 \%)$ & \\
Ovaria and uterus & $45(90 \%)$ & $5(10 \%)$ & \\
Omentum & $48(96 \%)$ & $2(4 \%)$ & \\
Biopsies $(>4)$ & $16(32 \%)$ & $34(68 \%)$ & \\
Douglas & $23(46 \%)$ & $27(54 \%)$ & \\
Bladder peritoneum & $32(64 \%)$ & $18(36 \%)$ & \\
Fossa ovarica right & $8(16 \%)$ & $42(84 \%)$ & \\
Fossa ovarica left & $9(18 \%)$ & $41(82 \%)$ & \\
Paracolic right & $37(74 \%)$ & $13(26 \%)$ & \\
Paracolic left & $37(74 \%)$ & $13(26 \%)$ & \\
diaphragm & $25(50 \%)$ & $25(50 \%)$ & \\
Lymph nodes $(\geq 10)$ & $34(68 \%)$ & $16(32 \%)$ & $12(38 \%)$ \\
Lymph nodes location & & & \\
$\quad$ - Aortocaval high & $25(50 \%)$ & $25(50 \%)$ & \\
- Pelvic ipsilateral & $41(82 \%)$ & $9(18 \%)$ & \\
- Pelvic contralateral & $25(50 \%)$ & $25(50 \%)$ & \\
Suspicious lesions & $30(60 \%)$ & $1(2 \%)$ & \\
Adhesions with tumor & $31(62 \%)$ & $7(14 \%)$ & \\
\hline & & & \\
& & & \\
& & & \\
\end{tabular}

6) comorbidity or malignancy elsewhere $(n=2)$, and 7) the wish of the patient to not receive adjuvant therapy $(n=1)$ (Fig. 2$)$.

In eight patients (16\%) no lymph nodes were resected, either because it was technically impossible $(n=4)$ or because the outcome of the lymph nodes, according to the gynecologist, had no therapeutic consequences ( $n=4$, Fig. 2 ). In 25 patients (50\%) no contralateral pelvic lymph nodes were resected. In eight patients (16\%) the number of resected lymph nodes was less than ten. This could be partly explained by the fact that in five of these patients only lymph nodes were resected on the ipsilateral pelvic region. Furthermore, although according to the gynecologist enough tissue was resected from the para-aortic region, the pathologist did not identify any lymph nodes in one patient (2\%). Taking ascites or peritoneal washing was omitted by accident in $12 \mathrm{pa}-$ tients (24\%), taking biopsies from adhesions surrounding the tumor was omitted in $14 \%$, an omentum biopsy or omentectomy in $2 \%$, and a biopsy of suspicious lesions in $2 \%$ of cases. A hysterectomy or contralateral ovariectomy was not performed in five cases (10\%) to preserve fertility or because of the wish of the patient to not receive adjuvant chemotherapy, and therefore a complete staging was not performed.

5-years survival. Out of the 50 patients included, 10 patients died within 5 years ( 5 -years survival $80 \%$ ). The number of missing elements was two in three patients, three in one patient, four in one patient, five in three patients, and six or more in the remaining two patients. In all patients either less than 10 lymph nodes and/or less than 3 locations was a missing element. In seven of these 10 patients the staging procedure was not completed because chemotherapy already being indicated based on peroperative findings. In the remaining three patients, despite an incomplete staging, no adjuvant chemotherapy was initiated. One patient, 58 years of age with a clearcell carcinoma, had a staging procedure in which less than five peritoneal biopsies were taken, and no pelvic lymph nodes on the contralateral side. This patient was offered adjuvant chemotherapy based on the pathological results (clearcell carcinoma), but she refused. The second patient, 25 years of age with a mucinous subtype carcinoma, underwent a second surgical procedure to complete the staging process in which, except leaving uterus and other normal-looking adnex in situ (fertility wish), no high para-aortic / paracaval lymph nodes were resected, and less than 5 peritoneal biopsies were taken. The third patient, 25 years of age with a mucinous carcinoma, also obtained a second surgical procedure to complete the staging process in which, except leaving uterus and other normallooking adnex in situ (fertility wish), no high para-aortal / paracaval lymph nodes were resected, and less than 5 peritoneal biopsies were taken. In this case, the surgeon took retroperitoneal tissue in the upper para-aortic and para-caval region, in which the pathologist however did not identify any lymph nodes.

\section{Discussion}

In this retrospective analysis we found that incomplete staging in EOC, performed by gynecologic oncologists, is most often due to insufficient biopsies of different locations of the peritoneum ( $n=34 ; 68 \%)$, and less than ten lymph nodes being resected and/or found at pathology ( $n=16 ; 32 \%$ ). The most mentioned reason for not performing biopsies was, besides forgetting to do so, believing that after careful inspection and palpation, taking blind biopsies is irrelevant and/or biopsies already are being taken while performing a hysterectomy (peritoneum of the cul-de-sac, broad ligament and bladder). Forgetting to take blind biopsies was registered in 11 cases and there can be little excuse to justify such negligence. Ten patients died within five years. Seven of these patients obtained adjuvant chemotherapy in addition to the staging procedure, and one patient refused adjuvant chemotherapy offered. The remaining two patients were young of age (both 25 years), and despite an incomplete staging with respect to both peritoneal biopsies and lymph nodes, no adjuvant chemotherapy was offered.

Previous studies report incidences of incomplete staging up to $98 \%$ [3,5-8]. In the majority of these patients no or inadequate lymph node resection was performed, but also no or an insufficient number of random peritoneal biopsies was frequently found $[3,8]$. Studies reporting on treated patients before 2000 conclude that lack of specialized skills and/or knowledge of the gynecologist might be a possible explanation for incomplete staging $[3,5,6,12]$. However, in the present study only gynecologic oncological center hospitals were included of which one can assume that they do have the skills and knowledge that is required. Nevertheless, taking blind biopsies was simply forgotten in about one third (11/34) of the incomplete biopsy cases. A standardized surgical report, and check lists with all parts of the staging procedure according to the FIGO guidelines available in the operation rooms, might be a solution to forgetting parts of the procedure.

It has been argued that upstaging of EOC on the basis of solely positive blind biopsies is low and therefore that taking blind biopsies is unnecessary. [8]. But this would be true for most of the different staging steps. In two reviews on surgical staging of EOC the average yield of positive blind biopsies of the peritoneum in a number of studies were found in 1.2 up to $9.3 \%$ [13,14]. From a more general perspective, it can be said that random peritoneal biopsies are recommended at an early stage of the disease, because it takes little extra time, entails almost no extra morbidity, and may result in a higher FIGO stage with thus therapeutic consequences. Furthermore, systematic peritoneal biopsies ensure careful palpation and examination of all surfaces. The latter notion is the more important in view of the recent trend to perform staging of EOC laparoscopically, therewith losing the contribution of palpating subperitoneal abnormalities [15].

Adequate lymph node sampling appears to be an essential part of the staging procedure in clinical early stage EOC. In a review on lymph node metastases, the overall incidence varied from $6.1 \%$ to $29.6 \%$ (mean 14.2\%) [16]. Despite its importance, the reported incidence of lymph node staging worldwide varies between $10 \%$ and $30 \%$ [17]. In a recent report on lymph node staging in The Netherlands, the incidence of lymph node dissection improved from 26\% in 2000 to 67\% in 2012. Moreover, the percentage of patients from whom 10 or more lymph nodes were removed also increased during the study period (from $2.3 \%$ to $47.6 \%$ ) [18]. In the present study, in 16 patients with an incomplete staging procedure (32\%), either no lymph nodes were resected or the number of lymph nodes identified was below ten. Various reasons for this were mentioned by the gynecologists. First of all, in five patients no lymph nodes were resected because it had no therapeutic 


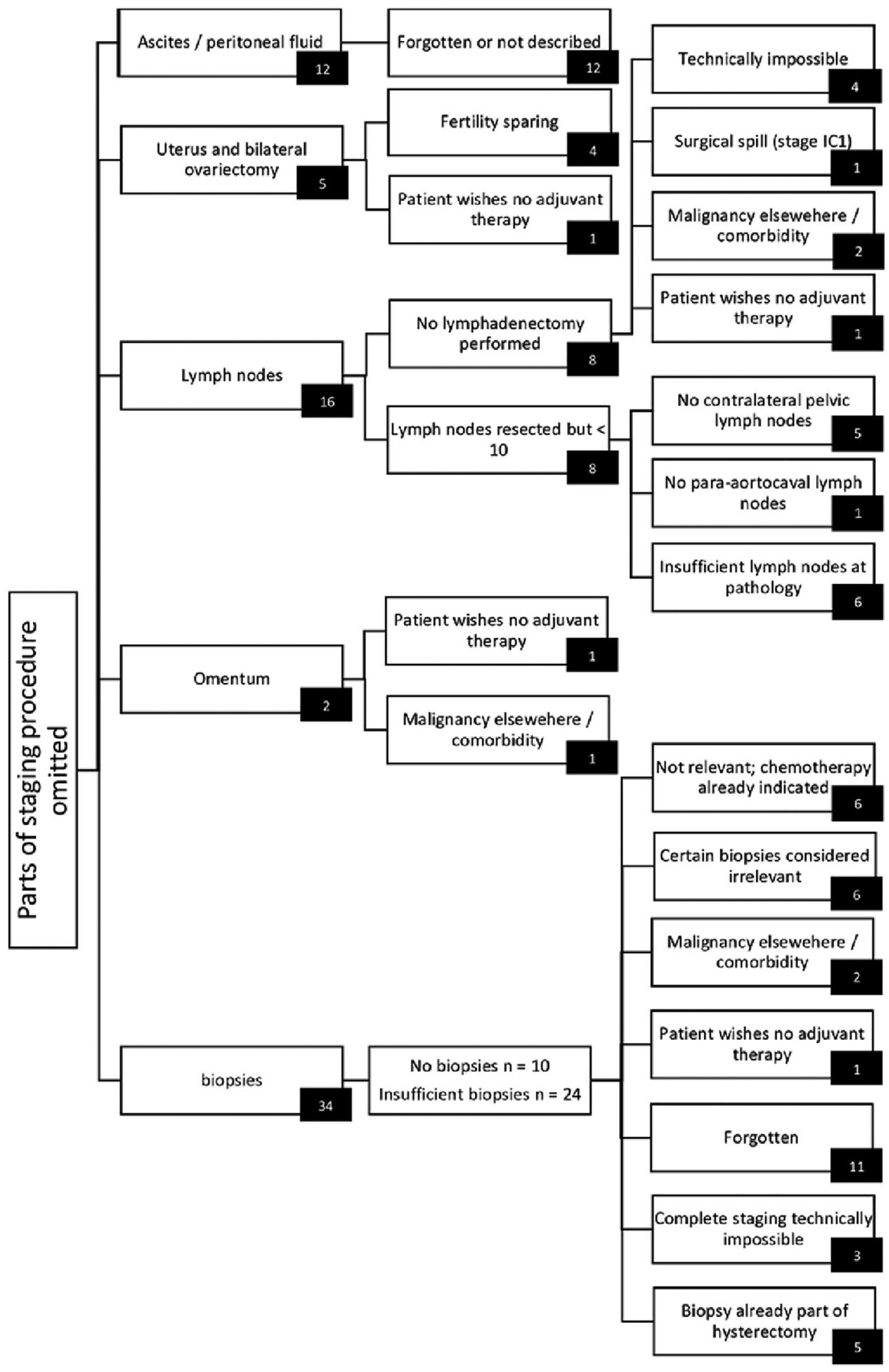

Fig. 2. Reasons for incomplete staging. Per staging element reasons for omitting the particular element of the staging procedure are described and counted.

consequences according to the gynecologist (already indication for adjuvant chemotherapy, i.e. the presence of rigid adhesions of the ovarian tumor to the pelvic wall clinically appropriate for FIGO stage IIB). In another four patients it was mentioned that the procedure was technically impossible, mainly related to intense intra-abdominal adhesions, extensive blood loss and/or obesity. Secondly, although according to the gynecologist enough tissue at the different pelvic and para-aortal locations were sampled, the number of lymph nodes identified by the 
pathologist were less than ten, hence insufficient according to the Dutch guideline. This could be partly explained by the fact that in five patients, pelvic lymph nodes were obtained only at the ipsilateral side. In $25 \mathrm{pa}-$ tients (50\%), including these five patients, the value of a contralateral pelvic lymphadenectomy in case of a unilateral ovarian tumor was questioned and therefore omitted. In the already mentioned review examining the incidence and locations of lymph node metastases in early stage EOC, $20 \%$ of all lymph node metastases were located at the pelvic regions only. Only contralateral lymph node metastases of a unilateral tumor were found in $16 \%$ of positive pelvic nodes and $11 \%$ of positive paraaortic nodes [16]. These findings provide a strong argument against an ipsilateral node dissection only. Current research into the feasibility of sentinel node detection in ovarian cancer would be a welcome alternative [19].

Besides the resection of not enough fatty tissue containing the lymph nodes by the gynecologist, the pathologist examining the tissue resected may influence the definite number of lymph nodes recognized. In a study observing the impact of nodal retrieval after educating the pathologist, it was found that extra education about the importance of nodal count significantly increased the nodal count at pathology [20]. New pathological methods are being investigated and have shown promising results. Svec et al. showed that re-fixing in a lymph node revealing solution containing ethanol, diethyl ether, glacial acetic acid and formalin, increased the number of revealed lymph nodes in colorectal resection specimens [21]. In another study comparing standard formalin fixation with fat-clearing by acetone in specimens after gastrectomy, more lymph nodes were found using acetone, though this was not statistically significant. More high-quality trials are needed to study the optimal technique for retrieving lymph nodes at pathology.

Complete staging has been proven to be an important prognostic factor in EOC $[4,18]$. There is evidence that, in contrast to incompletely staged patients, adjuvant chemotherapy in completely staged patients does not improve survival and should be omitted $[4,18,22,23]$. This argument makes a comprehensive and complete surgical staging the more important. We have analyzed the reasons for this and these data might serve to improve the staging performance of EOC. It should be said that the low percentages of complete surgical staging of EOC according to the existing guidelines might be flattered. In our study we found some reasons of the gynecologic oncologists for deliberately omitting certain staging steps in the individual clinical situation, perfectly plausible. In 34 patients the taking of blind peritoneal biopsies was incomplete. Of these, taking the biopsies was considered to have no therapeutic consequences in six patients, there were technical problems in three patients and comorbidity problems or a second malignancy in two. Together they represent one third of the incomplete biopsy group.

In 16 patients the yield of lymph nodes was incomplete with technical problems in four and no therapeutic consequences also in four. To convict these cases as victims of inadequate surgical performance would be unfair. For this reason, a plea can be made to separate cases such as these from the categories of complete and incomplete staging and refer to them as 'considerate adjustment of staging procedure'. It might be worthwhile to include this category in future studies on staging of EOC. In addition to this, future guidelines should leave more room for tailored procedures such as a stronger indication for surgical lymph node sampling (high grade serous tumors) and possible omission of sampling in case of low-grade mucinous histology.

In 2014, the Dutch Gynecological Oncology Audit (DGOA) was initiated to serve as a nationwide audit, which registers the four most prevalent gynecological malignancies [24]. Based on these data, benchmarked information based on quality indicators is given back to the user. At present, one of the quality indicators concerns completeness of surgical staging in clinical early-stage ovarian cancer. Such clinical auditing could be used as an instrument to improve care for patients with ovarian cancer.

The strength of this study is that by using the PRISMA methodology, we obtained detailed information regarding the reasons for incomplete staging. A limitation could be the time interval between the performed surgical procedure and the discussion with the surgeon based on the operation report, i.e. 1-5 years. But during the interviews this was never mentioned as a problem. Furthermore, it could be argued that in more recent years the completeness of surgical staging procedures may have improved. However, in a more recent publication with data from one of the Dutch gynecological oncology centers, the percentage of incomplete staging procedures was still 76.7\% [8].

\section{Conclusion}

The most common reasons for incomplete staging were insufficient random biopsies of the peritoneum, and less than ten lymph nodes being resected and/or found at pathology. The most mentioned reasons for not performing biopsies were forgetting to do so, or believing that, after careful inspection and palpation, taking biopsies is irrelevant and/or already are being taken while performing a hysterectomy (culde-sac, bladder). The value of contralateral pelvic lymph node dissection in case of a unilateral ovarian malignancy was also doubted, influencing the number of lymph nodes resected. Introducing a third staging category (considered adjustment of staging procedure) might give a more realistic picture of the actual staging performance. A structured list of staging steps to be used during surgery and evidence-based consensus of obligatory staging steps might help in achieving a higher rate of complete staging procedures.

\section{Credit author statement}

Laven P: Formal analysis, Investigation, Writing - Original Draft; Beltman JJ: Validation, Formal analysis, Investigation, Writing - Review \& Editing;

Bense JE: Formal analysis, Investigation, Writing - Original Draft;

van der Aa MA: Conceptualization, Methodology, Validation, Data Curation;

Van Gorp T: Validation, Writing - Review \& Editing;

Vos MC: Validation, Writing - Review \& Editing;

Boll D: Validation, Writing - Review \& Editing;

Arts HGJ: Validation, Writing - Review \& Editing;

Reesink N: Validation, Writing - Review \& Editing;

Trimbos JB: Validation, Writing - Review \& Editing;

Kruitwagen RFPM: Conceptualization, Methodology, Validation, Writing - Review \& Editing, Supervision.

\section{DECLARATIONS}

Author contributions. JJB, PL, JB, MA, MV, DB, HA, NR and JBT analyzing, reviewing and co-writing. MA selection of cases, analyzing and reviewing, RK design of study, analyzing, co-writing and reviewing. All authors have read and approved the manuscript.

Conflict of interest. The authors declare that they have no conflict of interest.

Funding. there was no funding for this study.

Ethics approval and consent to participate. The PRISMA commission consisted of eight gynecologists, a member of the NCR (MA), and a medical researcher. All included cases were analyzed and discussed with the gynecologic oncologists of each of the 5 participating hospitals. All members participating in the commission signed a non-disclosure agreement. Ethical approval was obtained in all regional hospitals (Commission of Medical Ethics, May 2015, reference number 15-4044). 


\section{References}

[1] Ledermann JA, Raja FA, Fotopoulou C, Gonzalez-Martin A, Colombo N, Sessa C. ESMO Guidelines Working Group. Newly diagnosed and relapsed epithelial ovarian carcinoma: ESMO Clinical Practice Guidelines for diagnosis, treatment and follow-up. Ann Oncol. 2013;24(Suppl 6):24-32.

[2] Benedet JL, Bender H, Jones 3rd H, Ngan HY, Pecorelli S. FIGO staging classifications and clinical practice guidelines in the management of gynecologic cancers. FIGO committee on gynecologic oncology. Int J Gynaecol Obstet. 2000;70:209-62.

[3] Timmers PJ, Zwinderman AH, Coens C, Vergote I, Trimbos JB. Understanding the problem of inadequately staging early ovarian cancer. Eur J Cancer. 2010;46:880-4.

[4] Zanetta G, Rota S, Chiari S, Bonazzi C, Bratina G, Torri V, et al. The accuracy of staging: an important prognostic determinator in stage I ovarian carcinoma. A multivariate analysis. Ann Oncol. 1998;9:1097-101.

[5] McGowan L, Lesher LP, Norris HJ, Barnett M. Misstaging of ovarian cancer. Obstet Gynecol. 1985;65:568-72.

[6] Trimbos JB, Schueler JA, van Lent M, Hermans J, Fleuren GJ. Reasons for incomplete surgical staging in early ovarian carcinoma. Gynecol Oncol. 1990;37:374-7.

[7] Sijmons EA, van Lankveld MA, Witteveen PO, Peeters PH, Koot VC, van Leeuwen JS. Compliance to clinical guidelines for early-stage epithelial ovarian cancer in relation to patient outcome. Eur J Obstet Gynecol Reprod Biol. 2007;131:203-8.

[8] Hengeveld EM, Zusterzeel PLM, Lajer H, Høgdall CK, Rosendahl M. The value of surgical staging in patients with apparent early stage epithelial ovarian carcinoma. Gynecol Oncol. 2019;154:308-13.

[9] Kaplan HS, Battles JB, Van der Schaaf TW, Shea CE, Mercer SQ. Identification and classification of the causes of events in transfusion medicine. Transfusion. 1998;38: 1071-81.

[10] Snijders C, van Lingen RA, van der Schaaf TW, Fetter WP, Molendijk HA, NEOSAFE study group. Incidents associated with mechanical ventilation and intravascular catheters in neonatal intensive care: exploration of the causes, severity and methods for prevention. Arch Dis Child Fetal Neonatal Ed. 2011;96:F121-6.

[11] Kasalak Ö, Yakar D, Dierckx RA, Kwee TC. Patient safety incidents in radiology: frequency and distribution of incident types. Acta Radiol. 2021;62:653-66.

[12] Soper JT, Johnson P, Johnson V, Berchuck A, Clarke-Pearson DL. Comprehensive restaging laparotomy in women with apparent early ovarian carcinoma. Obstet Gynecol. 1992;80:949-53.

[13] Trimbos JB. Surgical treatment of early-stage ovarian cancer. Best Pract Res Clin Obstet Gynaecol. 2017;41:60-70.
[14] Azaïs H, Estevez JP, Foucher P, Kerbage Y, Mordon S, Collinet P. Dealing with microscopic peritoneal metastases of epithelial ovarian cancer. A surgical challenge. Surg Oncol. 2017;26:46-52.

[15] Gallotta V, Jeong SY, Conte C, Trozzi R, Cappuccio S, Moroni R, et al. Minimally invasive surgical staging for early stage ovarian cancer: a long-term follow up. Eur J Surg Oncol. 2021. https://doi.org/10.1016/j.ejso.2021.01.033 S0748-7983(21)00063-9. Online ahead of print.

[16] Kleppe M, Wang T, Van Gorp T, Slangen BF, Kruse AJ, Kruitwagen RF. Lymph node metastasis in stages I and II ovarian cancer: a review. Gynecol Oncol. 2011;123: 610-4.

[17] Trope C, Kaern J. Adjuvant chemotherapy for early-stage ovarian cancer: review of the literature. J Clin Oncol. 2007;25:2909-20.

[18] Kleppe M, van der Aa MA, Van Gorp T, Slangen BF, Kruitwagen RF. The impact of lymph node dissection and adjuvant chemotherapy on survival: a nationwide cohort study of patients with clinical early-stage ovarian cancer. Eur J Cancer. 2016; 66:83-90.

[19] Dell'Orto F, Laven P, Delle Marchette M, Lambrechts Kruitwagen R, Buda A. Feasibility of sentinel lymph node mapping of the ovary: a systematic review. Gynecol Cancer. 2019;29:1209-15.

[20] Jeyarajah DR, Khithani A, Siripurapu V, Liu E, Thomas A, Saad AJ. Lymph node retrieval in pancreaticoduodenectomy specimens: does educating the pathologist matter? HPB (Oxford). 2014;16:263-6.

[21] Svec A, Horak L, Novotny J, Lysy P. Re-fixation in a lymph node revealing solution is a powerful method for identifying lymph nodes in colorectal resection specimens. Eur J Surg Oncol. 2006;32:426-9.

[22] Trimbos JB, Vergote I, Bolis G, Vermorken JB, Mangioni C, Madronal C, et al. Impact of adjuvant chemotherapy and surgical staging in early-stage ovarian carcinoma: European Organisation for Research and Treatment of Cancer-adjuvant ChemoTherapy in ovarian neoplasm trial. J Natl Cancer Inst. 2003;95:113-25.

[23] Trimbos JB, Timmer P, Pecorelli S, et al. Surgical staging and treatment of early ovarian cancer: long-term analysis from a randomized trial. J Natl Cancer Inst. 2010;102: 982-7.

[24] Baldewpersad Tewarie NMS, van Driel WJ, van Ham M, Wouters MW, Kruitwagen R. Participants of the Dutch gynecological oncology collaborator group. Clinical auditing as an instrument to improve care for patients with ovarian cancer: the Dutch gynecological oncology audit (DGOA). Eur J Surg Oncol. 2021;47:1691-7. 